\title{
Implikationen für eine partizipative politische Bildung
}

\subsection{Recht auf politische Partizipation in der Schule verankern}

Wenngleich die vorliegende Arbeit eine sozialwissenschaftliche Analyse ist und keinen verfassungs- oder schulrechtlichen Fokus hat, ist ein Blick auf die Verankerung von Partizipationsrechten der Schüler*innen dringend geboten, denn sie bildet die Grundlage für eine selbstbestimmte Beteiligung. Der Politikdidaktiker Reinhold Hedtke konstatiert, dass die „Erfahrung von echter, ernsthafter und effektiver politischer Partizipation [...] dem schulischen und außerschulischen Alltag der Schüler*innen fremd" (Hedtke 2020b, 140) bleibe. Wenn eine handlungsorientierte Politikdidaktik reale politische Handlungserfahrungen einbeziehen will, braucht es aber Freiräume in der Schule und im sozialen Nahraum, und diese dürfen nicht abhängig sein vom Wohlwollen der Lehrkräfte oder der Schulleitungen.

Aus den Schilderungen der interviewten Schüler*innen wird deutlich, dass sie die Schule nicht als einen Raum wahrnehmen, der ihnen adäquate Möglichkeiten zur Teilhabe schafft. Dass es sich dabei nicht nur um ein subjektives Gefühl Einzelner handelt, sondern sich auch durch repräsentative Befragungen bestätigt, zeigt unter anderem die Untersuchung „Kinder ohne Einfluss?“. Im Ergebnis zeigt die Studie deutlich: Schüler*innen fühlen sich auf allen Ebenen unzureichend beteiligt (Schneider u. a. 2009, 15-18). „In der Schule können Kinder nach eigenem Empfinden nur „wenig“ (60,4 Prozent) oder sogar „,̈̈berhaupt nicht“ (24,6 Prozent) mitbestimmen." (Stange 2010, 18). Das belegt auch eine aktuelle Studie für die Schüler*innen in Berlin befragt wurden: Die Schüler*innen geben mehrheitlich an, dass sie die Schule als einen Raum des offenen Meinungsaustausches erleben, sich aber nicht so stark involviert fühlen ,in Entscheidungsprozesse 
und in die tatsächliche Mitbestimmung an ihren Schulen“ (Achour u. a. 2020, 168).

Eine der hauptsächlichen Ursachen dafür, dass sich die Schüler*innen unzureichend beteiligt fühlen, liegt darin, dass institutionalisierte Partizipationsstrukturen noch immer viel zu häufig eher eine Scheinpartizipation (Schröder 1995, 16) darstellen und Schüler*innen in den etablierten Partizipationsstrukturen nur selten auf Augenhöhe die Interessen ihrer Peergroup vertreten können, sondern vielmehr Ordnungsaufgaben übernehmen müssen (siehe Abschnitt 2.1.3 „Kinder- und Jugendpartizipation“). Es braucht daher ein klares Bekenntnis in Form einer rechtlichen Verankerung zur selbstbestimmten Partizipation von Schüler*innen. Dafür ist auch eine ergebnisoffene Auseinandersetzung mit der Frage nötig, inwiefern Schüler*innenvertretungen ein allgemeinpolitisches Mandat haben dürfen. Das Interesse an allgemeinpolitischen Themen ist in den letzten 20 Jahren bei der jungen Generation gestiegen. Das zeigen Langzeitstudien wie die Shell-Jugendstudie (Albert u. a. 2019, 49). Gleichzeitig wird es der gewählten Interessenvertretung der Schüler*innen zumeist untersagt, sich zu allgemeinpolitischen Themen zu positionieren (siehe Abschnitt 2.3.1.2 „Politische Bildung und Partizipation rechtlich verankert?"). Dieser Widerspruch lässt sich kaum auflösen und es ist zu vermuten, dass dies auch dazu führt, dass sich politischer Protest von Schüler*innen und Studierenden immer häufiger in Form des zivilen Ungehorsams artikuliert. Solange die Schule sich einer Öffnung für den politischen Diskurs zu gesellschaftspolitischen Schlüsselproblemen verwehrt, werden sich Schüler*innen andere Möglichkeiten suchen müssen, um ihre Positionen in die öffentliche Debatte einzubringen. Dabei verliert die Schule immer wieder den Zugriff auf die damit verbundenen Bildungserfahrungen, aber auch die Möglichkeit Frustrationsmomente aufzufangen und potenziellen Radikalisierungstendenzen der Schüler*innen entgegenzuwirken.

Im Feld der formalen (politischen) Bildung besteht bis heute das Paradoxon, dass Schüler*innen zum selbstständigen und kritischen Analysieren, Urteilen und Handeln befähigt werden sollen, sie aber daran gehindert werden, als Konsequenz daraus Kritik an bestehenden Verhältnisse zu artikulieren und an deren Veränderung mitzuwirken. Mit einer Analogie lässt sich dieser Widerspruch verdeutlichen: Die Schule ist wie eine Sportstätte, in der Kinder und Jugendliche die grundsätzlichen Regeln und Techniken ihres Sports erlernen. Sie werden konfrontiert mit verschiedenen Trainingsmethoden und lernen taktische Konzeptionen kennen. Dabei bleibt ihnen aber die Möglichkeit verwehrt, die erlernten Fähigkeiten in der Praxis anzuwenden. Sie dürfen nicht mitspielen und ihre Ideen zur Weiterentwicklung nicht erproben. Das Ziel, kreativen Nachwuchs zu finden, 
der die Zukunft bestimmt, verbliebe in einem solchen Modell auf einer theoretischen Ebene. Für die Schule ist dieses Paradoxon verfassungs- und schulrechtlich festgeschrieben. So soll die Jugend zu einer „,politische[n] Verantwortlichkeit“ (Landesverfassung Baden-Württemberg, Artikel 12, Satz 1) und „zu freiheitlicher demokratischer Haltung" (Landesverfassung Sachsen, Artikel 101, Satz 1) erzogen werden, beruhend auf einem „Willen zu sozialer Gerechtigkeit“ (Landesverfassung Bremen, Artikel 26, Satz 1). Schulen sollen „nicht nur Wissen und Können vermitteln, sondern auch Herz und Charakter bilden“ (Landesverfassung Bayern, Artikel 131, Satz 1). Doch wie geht die Schule damit um, wenn Schüler*innen tatsächlich eine freiheitlich demokratische Haltung entwickeln, wenn sie Ungerechtigkeit wahrnehmen, ökonomische, ökologische und gesellschaftspolitische Missstände identifizieren? Wenn sie nicht nur ihre Fähigkeit in Bezug auf politisches Wissen und Können entwickelt haben, sondern als mündige Bürger*innen aus einer freiheitlich demokratischen Haltung heraus politisch aktiv werden wollen? Die Schule setzt dafür enge Grenzen und steht mit den zumeist unzulänglichen Mitbestimmungsrechten der Schüler*innen teilweise im Widerspruch zu den in den Landesverfassungen und Schulgesetzen formulierten Bildungszielen. So heißt es beispielweise in den Landesverfassungen von Brandenburg (Artikel 28) und Thüringen (Artikel 22), dass Erziehung und Bildung die Aufgabe haben, nicht nur „selbstständiges Denken“, sondern auch das „Handeln“ der Schüler*innen zu fördern.

In den meisten Bundesländern sind in Schulgesetzen die Partizipationsrechte von Schüler*innen nur über die etablierten Strukturen einer repräsentativen Interessenvertretung durch Klassensprecher*innen und der Schüler*innenvertretung geregelt. Wenngleich in wenigen Bundesländern wichtige Gremien wie die Schulkonferenz mit Drittelparität besetzt sind, so haben die Schüler*innen in den meisten Ländern und schulischen Gremien bis heute nur eine beratende Funktion oder ihre Stimme fällt kaum ins Gewicht. Teilweise hat die Schüler*innenvertretung sogar bis heute noch als verlängerter Arm der Lehrkräfte die Funktion, Ordnungsaufgaben zu übernehmen. Im Landesschulgesetz Bayern heißt es, dass zu den Aufgaben der Schüler*innenvertretung die „Übernahme von Ordnungsaufgaben [...] und die Mithilfe bei der Lösung von Konfliktfällen“ (Landesschulgesetz Bayern, Artikel 62, Satz 3) gehören. Die Instrumentalisierung der Schüler*innen in der Interessenvertretung ist damit rechtlich manifestiert.

Neben dieser offensichtlichen Limitierung der Partizipationsmöglichkeiten wird in den Schulgesetzen der Länder bis heute explizit oder implizit der Schüler*innenvertretung ein allgemeinpolitisches Mandat abgesprochen. Gemeint ist damit, dass sich Schüler*innenvertretungen nicht zu allgemeinpolitischen Themen positionieren dürfen. Eine direkte Ausformulierung dieses Verbots findet 
sich heute nur noch im Landesschulgesetz von Baden-Württemberg ( $\$ 63$, Absatz 3, Satz 3). Dort heißt es: „Klassenschülerversammlung und Schülervertreter haben kein politisches Mandat." Indirekt ist das Verbot eines allgemeinpolitischen Mandats aber noch in mehreren Landesverfassungen verankert, indem in einer Reihe von möglichen Aufgaben der Schüler*innenvertretung die politische Dimension explizit unerwähnt bleibt. Die Positionierung zu allgemeinpolitischen Themen wird ihnen nicht zugesprochen und ihr Wirkungsfeld auf den Schulbereich beschränkt. Das Land Berlin formuliert es im Schulgesetz ( $\$ 83$, Absatz 2) unmissverständlich: Die Aufgabe der Schüler*innen beziehe sich auf „bildungspolitische Fragen“.

Der Eindruck, dass die Schüler*innenvertretung unpolitisch sei, den mehrere interviewte Jugendliche formulierten, ist demnach nicht nur ein Gefühl, sondern eine verfassungs- und schulrechtliche Wirklichkeit. Die Beschränkung des Wirkungsfeldes der gewählten Interessenvertretung der Schüler*innen durch die Schulgesetze legitimiert allerdings keine Beschränkungen politischer Aktivitäten oder Positionierungen von Schüler*innen, die nicht für ein Amt gewählt wurden. Trotzdem zeigen die Praxis und auch die Schilderungen der für die vorliegende Untersuchung interviewten Schüler*innen, dass ihnen die Möglichkeit zur politischen Teilhabe im schulischen Umfeld immer wieder verwehrt wird. Dass es aber auch möglich ist, das Recht auf politisches Handeln im schulischen Kontext rechtlich zu verankern, zeigt das Fallbeispiel Niedersachsen. Hier ist mit dem $\S 86$ „Schülergruppen“ im niedersächsischen Schulgesetz das Recht für Schüler*innen formuliert, sich in Gruppen zu organisieren und für die durchaus allgemeinpolitischen Ziele einzustehen, die als Auftrag der Schule in $\S 2$ des Schulgesetzes formuliert sind. Hier heißt es unter anderem, dass die Schüler*innen befähigt werden sollen: zur „Erhaltung der Umwelt“ beizutragen, die „Grundrechte für sich und jeden anderen wirksam werden zu lassen“ und ,ihre Beziehungen zu anderen Menschen nach den Grundsätzen der Gerechtigkeit, der Solidarität und der Toleranz sowie der Gleichberechtigung der Geschlechter zu gestalten" (Schulgesetz Niedersachsen, § 2 Bildungsauftrag der Schule). Wollen sich Schüler*innen zur Erfüllung dieses Bildungsauftrages zusammenschließen, zum Beispiel eine Antirassismus-Arbeitsgemeinschaft gründen, ,so gestattet ihnen die Schulleiterin oder der Schulleiter die Benutzung von Schulanlagen und Einrichtungen der Schule" (Schulgesetz Niedersachsen, § 86 Schülergruppen). Diese Verankerung eines rechtlichen Anspruchs auf politische Partizipation in der Schule ist vorbildhaft.

Abschließend bleibt festzustellen, dass (politische) Partizipationsmöglichkeiten von Schüler*innen sowohl in den Landesverfassungen als auch in den Schulgesetzen bis heute eingeschränkt sind. Die politische Bildung kann über verbandspolitische Arbeit daraufhin hinwirken, dass sich daran etwas ändert. 
Damit Aktivitäten wie die derzeitige Initiative „Politische Bildung braucht Verfassungsrang“ (DVPB Niedersachsen 2020; Kenner 2020a, 41) des Landesverbandes Niedersachen der Deutschen Vereinigung für Politische Bildung (DVPB) nicht nur eine symbolische Wirkung haben, ist auch eine Bewusstseinsänderung bei Lehrkräften und Schulleitungen notwendig. Politische Selbstorganisation von Schüler*innen darf nicht als Gefährdung des Schulfriedens verstanden werden, unkonventionelle Partizipationsformen nicht als störend.

In der Schule wurde mir immer nur beigebracht, dass Protest nicht nötig ist, weil wir ja in der Demokratie leben und dass er andererseits nichts bringt, stressig ist, nervig irgendwie. Dass dadurch vielleicht der Schulbetrieb gestört wird, irgendwie und dass es unbequem ist. (FRANZISKA: 999-1004)

Erfahrungen dieser Art muss Schule etwas entgegensetzen. Politische Aktionen und reales politisches Handeln auch im Kontext der Schule sollten ermöglicht und als wertvolle Bildungsgelegenheit verstanden werden. Damit das gelingen kann, bedarf es aber mehr als nur rechtlicher Rahmenbedingungen. Politische Bildung kann und darf nicht neutral sein (siehe Abschnitt 2.3.1.1 „Politische Aktion und das vermeintliche ,Neutralitätsgebot?“"). Schule und Unterricht können für Kinder und Jugendliche wichtige Schutzräume für erste politische Handlungserfahrungen sein. So verweist SOPHIE darauf, dass sie bei ihrem rassismuskritischen Engagement an der Schule weniger Angst vor Übergriffen hatte als im Kontext außerschulischer politischer Aktionen:

\begin{abstract}
Aber das lag, glaube ich, auch an diesem Schulstatus, weil sonst bei anderen Aktionen, erinnere ich mich, war das irgendwie anders. Zum Beispiel bei Solidaritätsaktionen mit den Geflüchteten, wo teilweise nach irgendwelchen Nazi-Aufmärschen oder so, dann 20 Nazis zu den Geflüchteten gelaufen sind und die Leute zusammenschlagen wollten und wir da zu sechst standen und dachten: Okay, was machen wir jetzt? Aber das ist einfach ein ganz anderes Kaliber als ein Konzert von so einer Schule, glaube ich, das wird anders wahrgenommen. (KATJA: 364-375)
\end{abstract}

Die Probleme der Gegenwart beschäftigen Kinder und Jugendliche. Immer mehr junge Menschen wollen sich politisch positionieren und Einfluss nehmen. Wenn Schule den Zugriff auf diese Menschen nicht verlieren will, wenn politische Bildung die Erfahrungen in der politischen Aktion als wertvolle Lernanlässe aufgreifen will, muss Schule Freiräume schaffen, um sich mit diesen Themen auch losgelöst von didaktischen Konzeptionen im Politikunterricht befassen zu können und zugleich muss die formale politische Bildung die Bereitschaft zeigen, die damit verbundenen Bildungserfahrungen im Unterrichtssettings aufzugreifen. 


\subsection{Nicht neutral, aber... - Schule als politischen Lernort verstehen}

Die Schule wird von Schüler*innen als ein „Zweckverband zum Lernen“ (RONJA 213-213) wahrgenommen. Damit wird sie ihrem demokratischen Bildungsauftrag nicht gerecht. Gelingende emanzipative und partizipative politische Bildung als Didaktik sowie als Unterrichts- und Schulprinzip beruht auf einem angstfreien Bildungsraum, der es den Kindern und Jugendlichen ermöglicht, sich mit politischen Themen zu beschäftigen, Macht- und Herrschaftsverhältnisse zu analysieren, Fragen zu stellen, sich zu positionieren und - wenn gewollt - auch aktiv zu werden (Kenner 2018). Dieser Bildungsraum kann nur gemeinsam geschaffen werden, unter Einbeziehung aller beteiligten Akteur*innen, Schüler*innen, Lehrkräfte, dem pädagogischen und nicht-pädagogischen Personal sowie den Eltern. Er basiert auf wechselseitiger Zugewandtheit, Achtung und Respekt sowie Vertrauen und Zutrauen, aber auch auf dem Mut, Schüler*innen Gestaltungsmacht zu übertragen. Die vorliegende Untersuchung zeigt die Bedeutung dieses angstfreien Raumes. Vor allem Aussagen der Schüler*innen, die sich außerhalb der Schule selbstbestimmt politisch engagieren, lassen darauf schließen, dass sie die Schule nicht als einen solchen geschützten Raum erlebt haben.

Wenn Schule das Bildungsziel verfolgt, mündige Bürger*innen auf ihrem Weg zu begleiten, wenn der Auftrag schulischer Bildung auch darin bestehen soll, junge Menschen dazu zu befähigen, sich gesellschaftspolitischen Herausforderungen zu stellen und die Gesellschaft mitzugestalten, dann muss die Schule jenen, die schon in jungen Jahren die Bereitschaft zeigen, sich politisch zu engagieren, die Möglichkeit dazu bieten. LEA fasst diesen Wunsch wie folgt zusammen:

[M]an kann die Leute, die sich für Politik interessieren, lauter machen, indem man ihnen Gelegenheiten gibt, dass sie lauter werden können und dass sie sich selbst dazu entwickeln können, dass sie ihre Meinungen vertreten können. (LEA: 511-515)

Es gilt, das Politische als gleichwertigen und wesentlichen Bestandteil unserer Demokratie, genauso wie Kunst, Kultur und Sport, im schulischen Schutzraum zu etablieren. Bislang scheinen aber Sport und Musik als freiwillige Betätigungsfelder für Schüler*innen insgesamt eine höhere Anerkennung zu erfahren. So empfindet es auch RONJA (1006-1007): ,Sport oder Musik wird viel mehr akzeptiert als politisches Engagement." Wie die Fußball-Arbeitsgemeinschaft als Teil des schulischen Alltags sollte auch eine Antirassismus-Arbeitsgemeinschaft selbstverständlicher Bestandteil einer Schule sein können. Dieses Selbstverständnis als 
politischer Sozialisationsraum kann möglicherweise auch dazu beitragen die WirIhr-Konstruktionen politisch aktiver Schüler*innen (ausführlich Abschnitt 5.3.5.2) und damit verbundene Frustrationserfahrungen abzubauen. Dabei geht es nicht darum, dass Schüler*innen dazu angehalten werden, politisch aktiv zu sein, sondern darum, den interessierten Schüler*innen eine Infrastruktur zur Verfügung zu stellen und damit die Möglichkeit zu schaffen, sich politisch einzubringen. Auch LEA wünscht sich, dass sich mehr ihrer Mitschüler*innen politisch engagieren, aber sie ist entschieden dagegen, politisches Engagement als Zwang zu verordnen. „Es sollte von den Leuten selber kommen. “ (LEA: 495-498)

Grundlage für die Schule als Lernort der Demokratie (Kenner/Lange 2019) ist die Überzeugung aller Beteiligten, dass Schule die Selbstwirksamkeit der Kinder und Jugendlichen zu stärken hat. Eine hohe Selbstwirksamkeitseinschätzung ist Voraussetzung für eine gelingende Auseinandersetzung mit den Herausforderungen politischer und demokratischer Teilhabe. Für Schule als Lernort der Demokratie betont auch der Bildungswissenschaftler Wolfgang Edelstein, dass das psychologische Konstrukt der Selbstwirksamkeitsüberzeugung eine Voraussetzung für das Gelingen von Demokratisierungsprozessen in der Schule darstelle. Edelstein hebt hervor, dass die Überzeugung eigener Handlungsmächtigkeit „die Bereitschaft der Individuen zu einer Veränderung“ (Edelstein 2002, 18) durch das Vertrauen in die eigenen Kompetenzen bestärke. Eine Stärkung des politischen Selbstwirksamkeitskonzeptes der Kinder und Jugendlichen ist demnach Voraussetzung für eine demokratische Schule, die sich zum Ziel setzt, politische Handlungskompetenzen ihrer Schüler*innen zu stärken. Darüber hinaus ist die Haltung aller beteiligten Akteur*innen maßgeblich. Eine demokratische Grundhaltung, Dialogbereitschaft sowie Offenheit und Respekt gegenüber den Wahrnehmungen und Sichtweisen anderer - das sind wesentliche Bestandteile persönlicher Demokratiekompetenz (Eikel 2007, 25 ff.). Dafür müssen sich die demokratische Schule und insbesondere die Lehrkräfte von einer defizitorientierten Perspektive auf Kinder und Jugendliche lösen und sich dem Phänomen des Adultismus (Ritz 2013) verwehren. Schüler*innen sollten nicht unterschätzt, sondern unabhängig von Alter und Schulform in Aushandlungs- und Entscheidungsprozesse eingebunden werden. Es kommt der Schulentwicklung zugute, wenn unter den Beteiligten eine hohe Sensibilität gegenüber Macht-, Diskriminierungs- und Herrschaftsstrukturen besteht, sowie undemokratische Abläufe und Verhaltensweisen hinterfragt werden (siehe hierzu auch: Eis/Lösch u. a. 2015). Neugier, Frustrationstoleranz und Geduld sind dabei wichtige Grundhaltungen.

Freiräume zur Beteiligung in der Schule müssen immer wieder neu geschaffen, eingefordert und entwickelt werden. Neben den oben bereits beschriebenen Teilhabebarrieren gehört die Tatsache, dass ein Großteil der Schüler*innen nur 
unzureichend über ihre Teilhaberechte informiert ist, zu den zentralen Problemen. So haben in Niedersachsen, wie bereits erwähnt, Schüler*innen das Recht, politische Gruppen an der Schule zu gründen, in Berlin können Schüler*innen Vollversammlung einberufen. Vor allem die Vollversammlungen als eine Partizipationsform, die nicht orientiert am Einüben repräsentativdemokratischer Strukturen ist, sondern basisdemokratisches Handeln erfahrbar machen kann, wird im Alltag allerdings kaum umgesetzt (Kenner/Lange 2019, 129).

Schutzraum und Erprobungsfeld für politisches Handeln kann Schule nur werden, wenn Lehrkräfte das politische Handeln von Schüler*innen fachlich und pädagogisch begleiten. Das bedeutet nicht, dass sie politische Arbeitsgemeinschaften initiieren sollen. Vielmehr könnten sie jene Schüler*innen bestärken, die Interesse an politischer Selbstorganisation in der Schule signalisieren, und sie in dem Prozess begleiten. Das würde sich auch RONJA für ihr politisches Engagement wünschen: "[I]ch finde es schon auch hilfreich, wenn sie [die Lehrkräfte] dabeisitzen bei manchen Treffen einfach und ansprechbar sind." (RONJA: 492-495)

Schule ist ein wichtiger Sozialisationsort für Kinder und Jugendliche. Die Schule kann schon allein aus diesem Grund kein politisch neutraler Ort sein (siehe hierzu Abschnitt 2.3.1.1), sondern muss viel mehr gesellschaftspolitische Kontroversen der Gegenwart aufgreifen. Politische Bildung muss im Unterricht, aber auch in der Schule insgesamt dazu beitragen, dass marginalisierte Positionen und die Perspektiven der Ausgeschlossenen (de Moll u. a. 2013; Eis 2019; Kleinschmidt u. a. 2019) sichtbar gemacht werden. Die politischen Arbeitsgemeinschaften, die für die vorliegende Studie begleitet wurden, machen sich genau das zur Aufgabe. Eine wertvolle Bildungsgelegenheit, die es zu begleiten und zu fördern gilt. Und trotzdem müssen auch die Grenzen dessen, was multiperspektivisch und kontrovers im Kontext selbstbestimmter politischer Partizipation von Schüler*innen in die Schule getragen wird, festgelegt werden. Das sollte gemeinsam mit den Schüler*innen geschehen. Eine Möglichkeit wäre es, im pädagogischen Leitbild der Schule festzuhalten, dass sich die Schüler*innen in Arbeitsgemeinschaften zum Zwecke der Erfüllung des schulischen Bildungsauftrages politisch organisieren dürfen. Damit sind vielfältige politische Themenfelder wie unter anderem Antirassismus, Umweltengagement und soziale Gerechtigkeit eröffnet. Gleichzeitig kann im pädagogischen Leitbild auch das Selbstverständnis der Schule formuliert werden und damit eine klare Abgrenzung von jeder Form gruppenbezogener Menschenfeindlichkeit. Damit wäre der Rahmen für mögliches politisches Handeln von Schüler*innen in der Institution Schule gesteckt. 
Wer demokratische Einstellungen von Jugendlichen befördern will, muss [...] ihr demokratisches Handeln fördern. Dem sollte (auch) die Schule Raum geben und sich als erster Ort der Demokratie verstehen. Die Schule der Demokratie ist dann eine politische Schule. (Hedtke 2015, 125)

Der Politikdidaktiker Bernd Overwien sieht ein besonders hohes Potenzial in den Lernräumen, ,in denen sich formales und informelles Lernen treffen, in denen eigenständiges Lernen kulturell und sozial sensibel begleitet wird" (Overwien 2005, 352). Die Ermöglichung politischer Selbstorganisation der Schüler*innen in Verbindung mit einer pädagogischen Begleitung durch Lehrkräfte könnte ein möglicher Schritt dazu sein, diese Lernräume zu schaffen. Wenn das gelingt, bestehen beste Voraussetzungen für die Schule, nicht nur Lehranstalt, sondern auch Lernort der Demokratie zu sein.

\subsection{Von der Befähigung zur Erfahrung ${ }^{1}$}

Die Förderung der Handlungsfähigkeit ist weitgehend unbestritten eines der zentralen Ziele politischer Bildung und zugleich wohl das umstrittenste (Wohnig 2020b). Einigkeit besteht darin, dass alle Menschen durch politische Bildung dazu befähigt werden sollen, sich Informationen zu beschaffen, die Qualität von Quellen einzuordnen, gesellschaftspolitische Verhältnisse und Problemlagen zu analysieren und sich ein sachlich begründetes und wertgebundenes Urteil zu bilden. Weniger Einigkeit besteht darin, wie die politische Handlungsfähigkeit der Schüler*innen gestärkt werden kann.

Auf Grundlage der Erkenntnisse aus der vorliegenden empirischen Studie und der Rekonstruktion der Selbstbildungserfahrungen von Jugendlichen in selbstbestimmter politischer Aktion schlage ich vor Handlungsfähigkeit ins Zentrum pädagogischer und didaktischer Konzeptionen zu rücken und für die Schule auf drei Säulen aufzubauen. Die erste Säule beschreibt politisches Wissen über Entscheidungsprozesse und Handlungsstrategien sowie das kommunikative politische Handeln in didaktisierten und simulierten Lernsettings wie dem Talkshow-Format oder Planspielen (Petrik/Rappenglück 2017; Oberle/Leunig 2018). Sie helfen den Lernenden, sich in der Demokratie als Herrschaftsform zu orientieren und sich unabhängig von der eigenen Begeisterung für das jeweilige Themenfeld im politischen Argumentieren zu erproben. In der Politikdidaktik ist bis heute die Position etabliert, dass schulische politische Bildung über diese simulierten

\footnotetext{
${ }^{1}$ Teile der hier vorgeschlagenen Implikationen wurden in ähnlicher Form als Vorschlag zur Diskussion bereits in Kenner 2018 veröffentlicht und seither weiterentwickelt.
} 
Formate kommunikativer politischer Handlungen nicht hinausgehen sollte (siehe Abschnitt 2.2.2.7 „Handlungs- und Konfliktfähigkeit“ und 2.3.2 „Kontroverse: Partizipation als Ziel politischer Bildung?").

In den vergangenen Jahren mehren sich aber die Stimmen jener, die dieser Überzeugung etwas entgegensetzen wollen. Sie betonen dabei die Notwendigkeit des Blickes über den Tellerrand der politischen Bildung als Fachunterricht. Diese Perspektiverweiterung gelingt mithilfe der zweiten Säule: der demokratischen Schule. Wie von Vertreter*innen der Demokratiepädagogik (Beutel/Fauser 2007) gefordert, öffnet sich Schule als demokratischer Lernort im Nahbereich der Kinder und Jugendlichen, um Demokratie und Entscheidungsprozesse transparent und erfahrbar zu machen. Bezogen wird sich dabei vor allem auf die Überlegungen John Deweys zu „Demokratie und Erziehung“ (Dewey 1964). Das gleichnamige Buch erschien erstmals 1916. Hier skizziert er unter anderem die Wechselbeziehung von Erfahrung und Denken (Dewey 1964, 186-202). Alle Akteur*innen werden durch verschiedene methodische Zugänge wie das Lernen in Projekten (Juchler 2013; Koopmann 2005; Reinhardt 2007) und strukturelle Veränderungen wie die Einführung eines Klassenrates (Student/Portmann 2007) eingebunden (Beutel/Rademacher 2018; Edler 2018). Da die Mitwirkung aller Schüler*innen bei neuen Formaten der demokratischen Schulentwicklung vorausgesetzt wird, kann das Prinzip der Freiwilligkeit nur bedingt greifen (Kenner 2016, 17 f.). Daher ist die zweite Säule eher als Erprobungsfeld für soziales und demokratisches Handeln zu verstehen.

Bis hierhin wird deutlich, dass sich Grundprinzipien der Politikdidaktik und der Demokratiepädagogik nicht widersprechen müssen, sondern vielmehr gegenseitig befruchtende Konzepte demokratiebildnerischer Arbeit sind, die (fächerübergreifenden und fächerverbindenden) Unterricht und das demokratische Schulprinzip zusammendenken (Kenner 2020b, 119 f.). Neben den beiden hier benannten Grundpfeilern (1) „Wissen über und Simulation von Entscheidungsund Handlungsstrategien“ sowie (2) „Erfahrbarmachung demokratischen Handelns im Nahraum der Schule“ schaffte es die dritte Säule (3) „Ermöglichung von Selbstwirksamkeit durch selbstbestimmtes politisches Handeln“ nur am Rande in den Blick politikdidaktischer Forschung. Bei dieser dritten von mir vorgeschlagenen Säule stehen selbstbestimmtes und selbstorganisiertes Engagement sowie politische Partizipation als Bildungserfahrung im Fokus. Die Schule kann als Ermöglichungsraum politischer Selbstwirksamkeitserfahrungen fungieren, wobei dabei stets Freiwilligkeit und das Recht auf Nicht-Partizipation als Grundvoraussetzung gelten müssen (Nonnenmacher 2010, 466 f.).

Die dritte Säule einer handlungsorientierten politischen Bildung berücksichtigt politische Partizipations- und Artikulationsformen, die weder angeleitet noch 
eingefordert werden, sondern intrinsisch motiviert entstehen. Beispiele dafür sind unter anderem Antirassismus-Arbeitsgemeinschaften oder umweltpolitische Schüler*inneninitiativen. Diese dritte Säule macht für Schüler*innen, basierend auf den von Frank Nonnenmacher festgelegten differenzierten Kriterien für politische Bildung in Aktionen (Nonnenmacher 2010, 466), Politik erfahrbar. Politik und Demokratie würden mit der dritten Säule handlungsorientierter politischer Bildung nicht länger nur als Schulfach oder Prinzip einer demokratischen Schule wahrgenommen werden, sondern real erfahrbar gemacht.

„Die in der schulischen politischen Bildung vorherrschende Vorstellung einer Vorratsbildung für politisches Handeln in der Zukunft muss wohl vom Kopf auf die Füße gestellt werden.“ (Hedtke 2015, 125) Das kann mit einer handlungsorientierten politischen Bildung gelingen, die kommunikatives politisches Handeln in simulierten Settings schult, Raum für ernst gemeinte demokratische Teilhabe schafft und politische Partizipation ermöglicht. Die Erfahrungen, die dann mit den demokratischen Teilhabemöglichkeiten oder im Zuge realer politischer Partizipation und Selbstorganisation gesammelt werden, könnten dann vom Unterricht wieder aufgegriffen und in didaktischen Settings reflektiert werden. So wie Hermann Giesecke (1971) bereits in den 1970er-Jahren und Frank Nonnenmacher (1984) in den 1980er-Jahren vorgeschlagen haben, kann politische Aktion und politische Selbstorganisation der Schüler*innen dann zu einer wertvollen Bildungserfahrung werden, wenn sie in der Schule dafür den Freiraum bekommen und Aktion sowie Reflexion in einer ständigen Wechselbeziehung zueinander stehen.

Einer handlungsorientierten politischen Bildung gelingt es, neben den grundlegenden Kompetenzen wie Konfliktfähigkeit, Analysekompetenz und Urteilsbildung sowie Erfahrbarmachung demokratischer Handlungsstrategien auch Freiräume für tatsächliche politische Teilhabe zu schaffen und diese fachlich und pädagogisch zu begleiten, Anerkennung und politische Selbstwirksamkeit erfahrbar zu machen und Frustrationen aufzuarbeiten.

Um eine handlungsorientierte Didaktik der politischen Bildung zu etablieren, die diesem Anspruch gerecht wird, bedarf es struktureller Veränderungen an Schulen. Politische Bildung muss dafür Leitprinzip der demokratischen Schule werden, und es bedarf eines Bindeglieds zwischen formalen und informellen Bildungserfahrungen sowie zwischen schulischer und außerschulischer Bildung (Grüning 2020, 186; Wohnig 2018a) (Abbildung 5.1).

Wenn Mündigkeit als Ziel von politischer Bildung und demokratischer Schule insgesamt postuliert wird, so muss neu darüber nachgedacht werden, welche Rolle Handlungskompetenz im Prozess der Mündigkeitsbildung junger Menschen einnimmt. Eine handlungsorientierte politische Bildung sollte, wie oben beschrieben, 


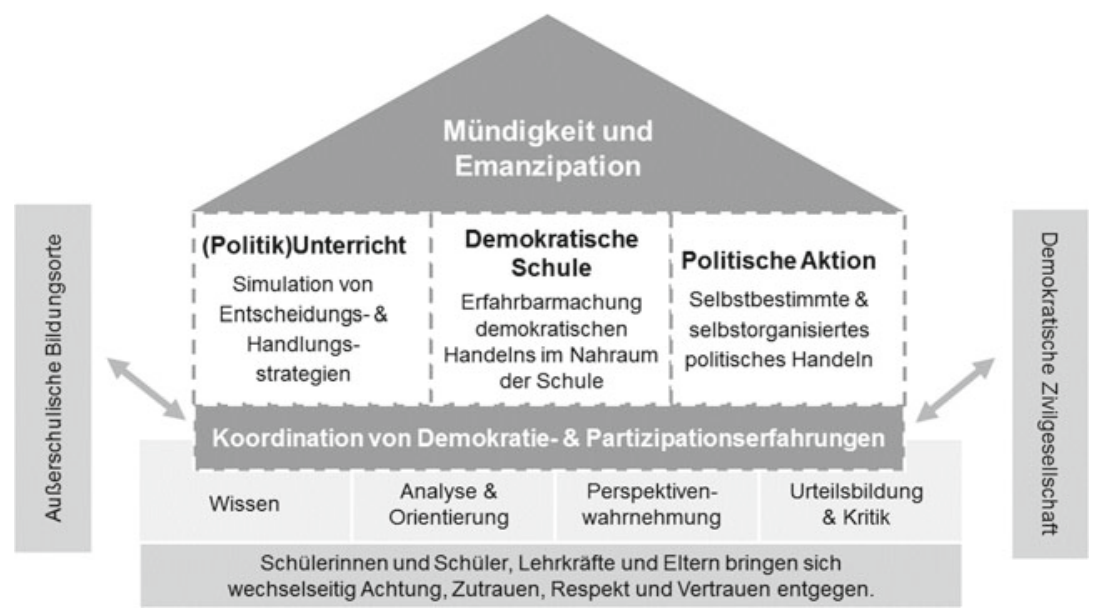

Abbildung 5.1 Partizipative und emanzipatorische politische Bildung in der Schule (eigene Darstellung)

auf drei Säulen aufbauen, die auf dem Fundament eines breiten Fachwissens und den grundlegenden Kompetenzen politischer Bildung - Analysekompetenz und politischer Orientierung, Konfliktfähigkeit sowie Urteilsbildung und Kritik - stehen:

1. Wissen über und Simulation von Entscheidungs- und Handlungsstrategien

2. Erfahrbarmachung demokratischen Handelns im Nahraum der Schule

3. Ermöglichung von Selbstwirksamkeit durch selbstbestimmtes politisches Handeln

Alle drei Säulen zusammen ermöglichen es, soziale und politische Teilhabeprozesse zu verstehen und innerhalb der Schule als Schutzraum Erfahrungen im Sinne einer jungen und emanzipatorischen Zivilgesellschaft zu sammeln. Damit Schule tatsächlich als Schutzraum wirken kann, müssen die Kinder und Jugendlichen pädagogisch und fachlich begleitet werden.

Um handlungsorientierte politische Bildung als Schulprinzip zu implementieren, muss sich der Stellenwert dieser bildungspolitischen Aufgabe entsprechend des Bildungsauftrages der Schule auch in den strukturellen Gegebenheiten der Schule widerspiegeln. Es bedarf einer Koordinierung der verschiedenen politischen Bildungsgelegenheiten und Erfahrungsräume. Diese Koordinierung müsste 
nicht zwingend von Lehrkräften vorgenommen werden. Vielmehr sollte die Infrastruktur und die Voraussetzungen für einen Bottom-up-Prozess geschaffen werden.

Planspiele, Inselexperimente, Schüler*innenparlamente, Vollversammlungen, Klassenräte, politische Arbeitsgemeinschaften gegen Rassismus, Aktionstage gegen Sexismus und Homophobie, Flüchtlingskonferenzen, Unterschriftenaktionen gegen die neue Handyregelung an der Schule - all diese Partizipationsformate stehen beispielhaft für eine gelebte Demokratie und eine handlungsorientierte politische Bildung. Die vorliegende Studie bestätigt die von Andreas Eis formulierte These, dass sich kollektive politische Bildungsprozesse in Handlungssituationen vollziehen, ,in denen nicht in einem top-down-Verfahren Scheinprobleme und pseudopartizipatorische Entscheidungs- und Lernanlässe vorgegeben werden, sondern die Subjekte auf eigene Handlungsproblematiken des gesellschaftlichen Zusammenlebens stoßen und ihre politische Selbst- und Weltverfügung lernend erweitern" (Eis 2014, 266). Dafür sollte die Schule als Ort politischer Sozialisation Freiräume schaffen.

Die Öffnung für reale politische Partizipationsprojekte kann an der Schule aber auch zu Kontroversen führen. Doch warum sollte ausgerechnet die Schule ein Ort sein, an dem gesellschaftliche Kontroversen nur - wenn überhaupt - künstlich, fiktiv und imaginär durch abstrakte Unterrichtskonzepte oder simuliertes politisches Handeln in Rollen- und Planspielen erfahrbar werden? Die Kontroverse und der politische Konflikt gehören zur Demokratie. Die für die vorliegende Studie interviewten Schüler*innen haben das erkannt.

Wenn eine handlungsorientierte politische Bildung in der Schule umgesetzt werden soll, müssen für alle Beteiligten die Grundsätze des Überwältigungsverbots und des Kontroversitätsgebots (Wehling 1977) gelten, ohne dass sie dabei wie ein Damoklesschwert über allen schweben (Kenner 2016, 17). Sie müssen als Orientierung und Leitplanken dienen, aber dürfen nicht als vorgeschobene Rechtfertigung einer instruktionsorientierten und institutionenkundlichen politischen Bildung dienen, der es an Mut fehlt, Politik und Demokratie in der Schule erfahrbar zu machen. Andreas Eis, Bettina Lösch, Achim Schröder und Gerd Steffens haben die Prinzipien der politischen Bildung, die im sogenannten „Beutelsbacher Konsens" formuliert sind, um wesentliche Aspekte erweitert und dies in der „Frankfurter Erklärung“ zusammengetragen. Sie betonen dabei den Wert des individuellen und kollektiven Handelns für eine kritisch-emanzipatorische politische Bildung (Eis/Lösch u. a. 2015; Eis 2016). Die Prinzipien des „Beutelsbacher Konsenses“ in Verbindung mit der „Frankfurter Erklärung“ könnten eine differenzierte Grundlage für eine handlungsorientierte politische Bildung an Schulen sein und neben den etablierten Kompetenzfeldern der politischen Bildung vor allem 
auch die Fähigkeit zum kritisch-emanzipatorischen Denken, die Stärkung realer politischer Handlungsfähigkeit und damit verbunden auch die Befähigung zum Widerspruch und Widerstand in den Fokus rücken.

Open Access Dieses Kapitel wird unter der Creative Commons Namensnennung 4.0 International Lizenz (http://creativecommons.org/licenses/by/4.0/deed.de) veröffentlicht, welche die Nutzung, Vervielfältigung, Bearbeitung, Verbreitung und Wiedergabe in jeglichem Medium und Format erlaubt, sofern Sie den/die ursprünglichen Autor(en) und die Quelle ordnungsgemäß nennen, einen Link zur Creative Commons Lizenz beifügen und angeben, ob Änderungen vorgenommen wurden.

Die in diesem Kapitel enthaltenen Bilder und sonstiges Drittmaterial unterliegen ebenfalls der genannten Creative Commons Lizenz, sofern sich aus der Abbildungslegende nichts anderes ergibt. Sofern das betreffende Material nicht unter der genannten Creative Commons Lizenz steht und die betreffende Handlung nicht nach gesetzlichen Vorschriften erlaubt ist, ist für die oben aufgeführten Weiterverwendungen des Materials die Einwilligung des jeweiligen Rechteinhabers einzuholen.

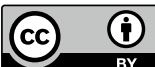

